\title{
Die Leiden des Charles Darwin
}

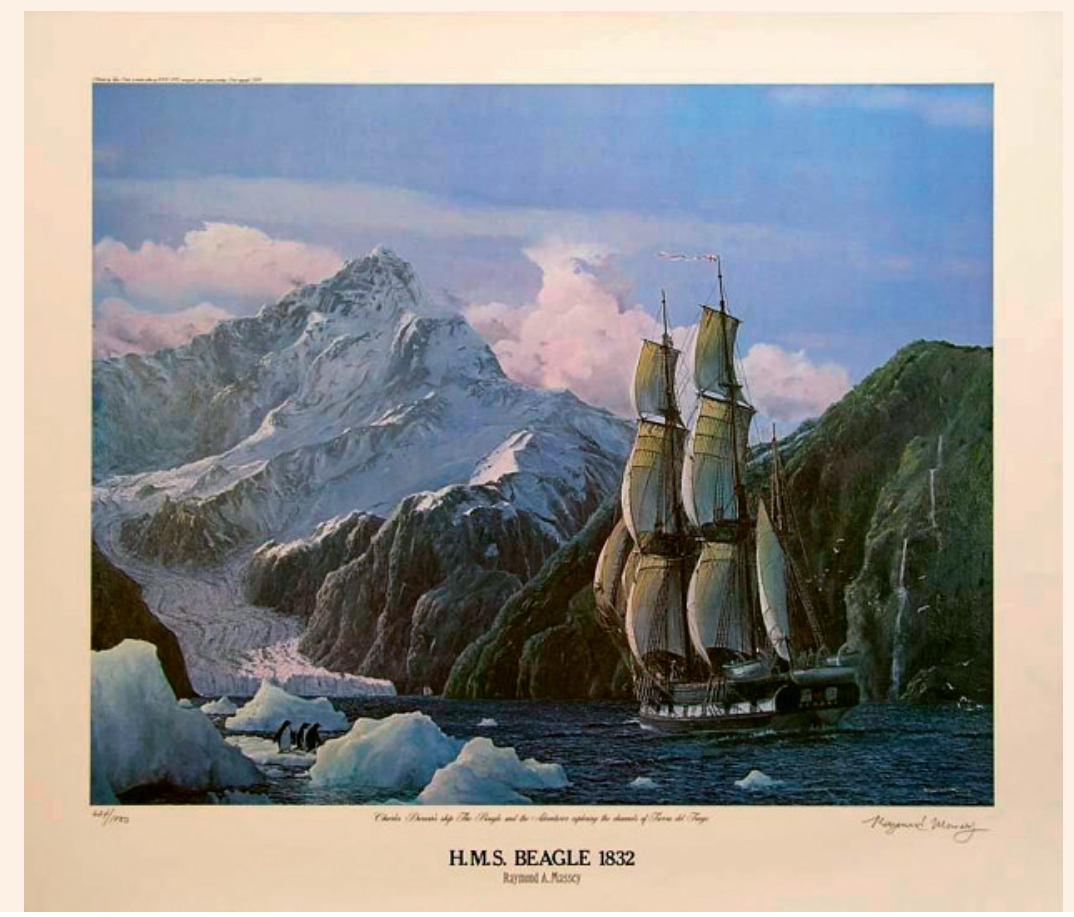

Ronald D. Gerste

Korrespondenz:

Dr. med. Ronald D. Gerste 14801 Soft Wind Drive

USA-Gaithersburg, MD 20878

rdgerste@aol.com
Dem Mann ging es nicht gut - wieder einmal. «Alles, was mich aufregt, wirft mich vollständig um und führt zu schmerzhaften Palpitationen des Herzens», wie er seinem mit Akribie geführten Tagebuch anvertraute, das über viele Seiten einer Leidensgeschichte gleicht und mit einem erstaunlichen Artenreichtum von Beschwerden angefüllt ist: «Der Kopf schwimmt mir, Depression, Zittern - vielfältige Attacken der Krankheit.» Dazu: «viel unabänderliche Angst» wegen des Herzrasens, das ihm in besonders dunklen Stunden sein baldiges Ende anzukündigen schien. Vielfältige Symptome, die ihn oft tagein, tagaus plagten - und die ihn dennoch nicht an der Fertigstellung seines Lebenswerkes hinderten, das zu den elementarsten, um nicht zu sagen: revolutionärsten, in der Wissenschaftsgeschichte gehört, einem Euvre, das ihn auf eine Stufe mit Kopernikus und Einstein stellt: der Vielleidende war der grosse Naturforscher Charles Darwin.

Mit Charles Darwin wird man in diesem Jahr auf vielfältige Weise konfrontiert - Grund ist der 200. Geburtstag des Begründers der modernen Evolutionslehre. Darwin kam am 12. Februar 1809 zur Welt; wie es der Zufall will, an exakt dem gleichen Tag wie ein anderer Grosser der Weltgeschichte, der amerikanische Präsident und Sklavenbefreier Abraham Lincoln. Darwins Werk ist eine Grundlage modernen Naturverständnisses. Unumstritten ist es nicht, da viele gläubige Christen in seiner Lehre eine Attacke auf die Bibel und letztendlich gar auf Gott selbst sehen. Mit dieser Ablehnung seines Werkes hatte Darwin bereits zu Lebzeiten zu kämpfen. Es war ein Kampf, der vor seinem engsten Umfeld nicht Halt machte. Seine Frau Emma war tief religiös und hat ihren Gatten mit seinen Erkenntnissen möglicherweise auf dem Weg zur Blasphemie gesehen. Hat dieser Konflikt mit dazu beigetragen, Darwin krank zu machen?

Denn Krankheit ist ein unauslöschlicher Bestandteil des Lebensweges von Charles Darwin und gleichzeitig ein Rätsel, das seine zahlreichen Biographen zu Hypothesen angeregt hat; ein Rätsel, das indes nie gelöst werden konnte. Man will nicht einmal mit letzter Sicherheit ausschliessen, dass Darwin sich die Symptome - von rasenden Kopfschmerzen über Sehstörungen bis zu den von ihm ziemlich detailliert beschriebenen Brechattacken - regelrecht «zugelegt» hat, um seine sozialen Interaktionen auf ein Minimum zu begrenzen, sich zeitweise wie ein Eremit abzukapseln und dabei die dringend benötigte Zeit und Ruhe zu finden, um sein monumentales Werk The Origin of Species by Means of Natural Selection (Die Entstehung der Arten durch natürliche Zuchtwahl) zur Reife kommen zu lassen. Und vor allem Zeit brauchte Charles Darwin im Übermass: Vom Beginn seiner Weltreise mit der Beagle, auf welcher die Grundlagen seiner Ideen gesammelt wurden, bis zur Erstveröffentlichung der Origin of Species vergingen nicht weniger als 28 Jahre.

Der intensiven Auseinandersetzung mit den eigenen zum Teil sicher realen, in manchen Fällen aber möglicherweise aggravierten Krankheitssymptomen war es zweifellos hochgradig förder- 
lich, dass Charles Darwin an jenem 12. Februar 1809 in eine Arztfamilie hineingeboren wurde. Sein Grossvater, Dr. Erasmus Darwin, gilt als eine der grössten britischen Medizinerpersönlichkeiten des 18. Jahrhunderts. In dessen Fussstapfen trat auch der Sohn, Charles Darwins Vater, Dr.
Darwin selbst diesen Exkurs «spasshaft» und resümierte, dass die halbherzige Berufswahl, die eines anglikanischen Geistlichen, «eines natürlichen Todes gestorben [ist], als ich beim Verlassen von Cambridge als Naturforscher an Bord der Beagle ging.»

\section{«Mein Vater erklärte, dass ich ein erfolgreicher Arzt werden solle - das heisst: einer, der viele Patienten hat.»}

Robert Waring Darwin. Dieser lebte und praktizierte in Shrewsbury, seine Praxis soll von Reputation, Patientennachfrage und Umsatz eine der grössten des Königreiches ausserhalb von London gewesen sein. Die Prosperität des Dr. Darwin spiegelt Darwins Geburtshaus wider. Das «The Mount» genannte Domizil der Arztfamilie kann man getrost eher ein Anwesen, ein Palais denn ein Einfamilienhaus nennen. Den erfolgreichen und angesehenen Vater betrachtete der junge Charles Darwin mit unverhohlener Bewunderung: «Mein Vater, welcher der weiseste Mann gewesen ist, den ich je gekannt habe [...].» Möglicherweise hat das Bewusstsein, sich in einem übermächtigen Schatten bewähren und aus diesem heraustreten zu müssen, eine Konfliktsituation bei Charles ausgelöst, die zu seinen somatischen Beschwerden erheblich beigetragen haben mag.

Und ganz ohne Frage wurde von Seiten des Vaters beträchtlicher Druck auf Charles Darwin ausgeübt, als es um die Frage der Berufswahl ging. Für Darwin senior kam nur eine Möglichkeit in Frage: «Mein Vater erklärte, dass ich ein erfolgreicher Arzt werden solle - das heisst: einer, der viele Patienten hat.» Folgsam, wie Charles war, immatrikulierte er sich 1825 - also als 16-Jähriger! - an der Medizinischen Fakultät der Universität Edinburgh, damals in der Welt der akademischen Heilkunde eine der besten Adressen. Doch das Studium begeisterte ihn nicht. Die Vorlesungen empfand er als langweilig, zwei Operationen, an denen er - 20 Jahre vor Entdeckung der Anästhesie - als Zuschauer teilnahm, entsetzten ihn zutiefst: «Ich aber lief davon, ehe sie zu Ende gebracht waren.» Die operative Medizin empfand er als beastly profession, im zweiten Jahr an der Universität der schottischen Hauptstadt belegte er zunehmend Vorlesungen in Zoologie und Geologie, die ihm auf seinem späteren Lebensweg nützlicher waren als Kurse in Practice of Physic (eine Art klinischer Untersuchungskurs) und Midwifery (Geburtshilfe). Er begann dann in Cambridge das Studium der Theologie, doch auch dies vollendete er nicht. Viele Jahre später nannte
Die Fahrt mit der Beagle wurde zum entscheidenden Erlebnis für den jungen Darwin, dem das Angebot gemacht wurde, das kleine Schiff auf einer primär der Region um Feuerland gewidmeten Vermessungsmission als Naturforscher zu begleiten. Die Reise dauerte fast fünf Jahre, vom Dezember 1831 bis zum Oktober 1836. Darwin erkundete dabei unter anderem den Dschungel Brasiliens, die Pampas Argentiniens, die grandiosen Küstenlandschaften Südamerikas, die Gebirgszüge der Anden, vor allem aber - bevor es auf die lange Heimreise über Australien, Mauritius und Südafrika ging: die Galapagos-Inseln. Hier fielen ihm zahlreiche Besonderheiten der Schöpfung, der Natur oder der - wie es aufgrund seines Wirkens bald heissen sollte - der Evolution auf, die so höchst eigentümlich waren, dass er nach einer Erklärung für ihre Vielfalt zu suchen begann. Ein wahres Damaskuserlebnis waren für ihn die Beobachtungen des Artenwandels innerhalb bestimmter Vogelarten, vor allem einer «äusserst eigentümlichen Gruppe von Finken, die in der Struktur ihrer Schnäbel, den kurzen Schwingen, der Form des Körpers und dem Gefieder miteinander verwandt sind. Es sind dreizehn Spezies und ... alle Spezies sind diesem Archipel eigentümlich.» Seine Beobachtungen, Notizen und Zeichnungen sollten sich als Stoff für eine sich über Jahrzehnte erstreckende wissenschaftliche Arbeit erweisen, nach deren Publikation das Verständnis des Menschen von der Natur nicht wieder so sein sollte wie vor Charles Darwin.

Doch auch diese grandiose wissenschaftliche Reise schien stets bedroht von Darwins angegriffener - oder subjektiv als angegriffen empfundener Gesundheit. Schon am Anfang der Exkursion hing die Sorge um die eigene, als höchst unzulänglich betrachtete Gesundheit wie ein Damoklesschwert über dem Forscherdrang des Charles Darwin. So sah er sich als kardiologisch schwer affektiert: «Ich wurde durch Herzklopfen und Schmerzen in der Herzgegend beunruhigt und war wie so viele unwissende junge Leute, besonders wie solche mit oberflächlichen medi- 
zinischen Kenntnissen, überzeugt, dass ich einen Herzfehler habe. Ich habe keinen Arzt konsultiert, da ich vollständig überzeugt war, von ihm hören zu müssen, dass ich zur Reise untauglich sei, und doch war ich entschlossen, unter allen Umständen zu gehen.»Wie nicht anders zu erwarten, wurde er auf der Beagle wiederholt seekrank. Doch auch bei den sich oft über Wochen erstreckenden Landausflügen schlug das Schicksal, glaubt man seinen Tagebucheintragungen, immer wieder unerbittlich zu. Eintragungen wie «extrem ermüdet», «äusserst schwach», «unwohl und fiebernd» wechselten sich mit der Standardselbstbeobachtung quite unwell in unschöner Regelmässigkeit $a b$.

Die ausführliche Dokumentation der Eigenanamnese nahm nach seiner Rückkehr nach London und ab 1842 nach der Übersiedlung in den kleinen Ort Downe in der Grafschaft Kent fast epische Ausmasse an. Kaum hatte er wieder englischen Boden unter den Füssen, da verspürte er

\section{Ich habe keinen Arzt konsultiert, da ich vollständig überzeugt war, von ihm hören zu müssen, dass ich zur}

\section{Reise untauglich sei}

1 Colp R. To Be an Invalid. The Illness of Charles Darwin. Chicago: University Press; 1977.

2 Darwin C. Mein Leben 1809-1882. Vollständige Ausgabe der «Autobiographie». Frankfurt: Insel; 2008.

3 Hemleben J. Charles Darwin. Mit Selbstzeugnissen und Bilddokumenten. 14. Auflage. Reinbek: Rowohlt; 2004. ernde Gefährten (Freunde im hohen Alter), die sich für uns interessieren und die wir lieben und mit welchen wir spielen, sicherlich besser als ein Hund. Ein Heim und jemand, der das Haus besorgt. Das Anziehende von Musik und weiblichem Geplauder.» Diese Aufzählung kulminierte in der höchstmöglichen Gewinnerwartung: «Diese Dinge sind gut für die Gesundheit.» Dem standen an Nachteilen gegenüber: «Schrecklicher Zeitverlust und, wenn viele Kinder kommen, gezwungen zu sein, sein Brot zu verdienen.» Sein Brot zu verdienen, war er in der Tat gezwungen - dem Ehepaar Darwin wurden zehn Kinder beschert.

$\mathrm{Zu}$ Herzpalpitationen und zentralnervösen Erscheinungen wie Kopfschmerzen, Verschwommensehen und Schwindel traten in den folgenden Jahren vor allem Probleme des Gastrointestinaltraktes. Die detaillierten Schilderungen des Leidenden von der Geschmacksqualität seines Erbrochenen und der Ausprägung seiner Flatulenz seien dem Leser und der Leserin an dieser Stelle erspart. Rund zwanzig Ärzte sollen sich im Laufe seines Lebens seines meist nur vorübergehenden Vertrauens erfreut haben; vor allem in recht obskure Wasserkuren setzte er immer wieder grosse Hoffnungen. Als die Veröffentlichung von Origin of Species im November 1859 kurz bevorstand - die erste Auflage von 1250 Exemplaren war übrigens binnen weniger Stunden ausverkauft - traf es ihn, der zu diesem Zeitpunkt zweifellos unter hoher emotionaler Anspannung stand, besonders heftig. Der ihn zu diesem Zeitpunkt behandelnde Arzt bekam seine reduzierte Grundstimmung zu spüren: «Alle sagen, dass Dr. Smith bei schweren Krankheiten sehr gewissenhaft sei, aber er gibt mir permanent den Eindruck, als sorge er sich sehr um sein Honorar und nur sehr wenig um seinen Patienten.»

Darwins Gesundheitszustand sollte sich in seinem sechsten und siebten Lebensjahrzehnt erstaunlicherweise verbessern - offenbar parallel zu seinem gestiegenen gesellschaftlichen Ansehen, nachdem sein Werk zunehmend akzeptiert wurde. Erst in der Endphase seines Lebens scheint ein Herzleiden wirklich manifest gewesen zu sein. Der Sohn des ihn zuletzt behandelnden Arztes schrieb später, «mein Vater berichtete mir, dass er Zeichen einer myokardialen Degeneration bei Darwin festgestellt hatte». Angina pectoris ist vielleicht die einzige gesicherte Diagnose bei diesem Patienten, dem in den letzten Lebenswochen wiederholt Amylnitrit verabreicht wurde. Charles Darwin starb am 19. April 1882, im Alter von 73 Jahren, und wurde unter grosser Anteilnahme der Öffentlichkeit in der Westmins-

ter Abbey beigesetzt. 